\title{
The effectiveness of using deep learning algorithms in predicting students achievements
}

\author{
Mohammed Akour ${ }^{1}$, Hiba Al Sghaier ${ }^{2}$, Osama Al Qasem \\ ${ }^{1}$ Al Yamamah University, Saudi Arabia \\ ${ }^{1,2,3}$ Yarmouk University, Jordan
}

\begin{tabular}{l} 
Article Info \\
\hline Article history: \\
Received Oct 13, 2019 \\
Revised Dec 1, 2019 \\
Accepted Dec 11, 2019 \\
\hline
\end{tabular}

\begin{abstract}
Educational Data Mining (EDM) research has taking an important place as it helps in exposing useful knowledge from educational data sets to be employed and serve several purposes such as predicting students' achievements. Predicting student's achievements might be useful for building and adopting several changes in the educational environments as a re-action in the current educational systems. Most of the existing research have used machine learning to predict students' achievements by using diverse attributes such as family income, students gender, students absence and level etc. In this paper, the effort is made to explore the effectiveness of using the deep learning algorithm more precisely CNN to predict students' achievements which could help in predicting if student will be able to finish their degree or not. The experimental results reveal how the proposed model outperformed the existing approaches in terms of prediction accuracy.
\end{abstract}

Copyright $\odot 2020$ Institute of Advanced Engineering and Science. All rights reserved.

\section{Corresponding Author:}

Mohammed Akour,

Department of Computer Engineering,

AL Yamamah University,

Riyadh, Saudi Arabia.

Email: m_akour@yu.edu.sa

\section{INTRODUCTION}

Educational institutions are running in an increasingly competitive and complex atmosphere, and facing a burden in dealing with global and national economic, these institutions try to make change such as the growing need to develop the ratio of students in specific disciplines, and ensuring that the quality of learning programs are both globally and nationally relevant [1].

Students are the primary stakeholders in the educational institutions and their performance play a noteworthy role in a nation's social and monetary development by delivering imaginative alumni. There is a basic interest for scholarly establishments to keep up and to incorporate enormous datasets of students for multipurpose basic leadership. The utilization of web innovation has additionally turned into integral part of the current era of education in many universities, expanding the real measure of information about students, instructors and their communications with learning and instructive frameworks. Advanced education play a significant role in the improvement and development of a society. It is a field, which gives a lot of information about members, for example, students, instructors, offices and educational programs. The performance of students is a principle concern of different partners including teachers, overseers and organizations. Therefore, students have to work hard for excellent grades, so that they may rise up to the expectations of education partners [2].

Student's performance is a fundamental factor in educational institutions, as the excellent academic achievement of specific university will lead to improving the quality of that university. Student's performance can be acquired by measuring the 1 co-curriculum and earning assessment. However, most of the researches mentioned that graduation is the measure of student's success [3]. 
Data science protrudes as a new discipline. It is viewed as an integration of classical disciplines like data mining, statistics, distributed systems and databases. Current approaches need to be combined to turn abundantly available data into valuable information for society, individuals and organizations. Data science techniques can be used to discover processes, analyze bottlenecks, and check compliance and even to suggest improvements [4].

Data science depends on deriving knowledge, insights, and predictive models from raw data. This attempt includes curating and cleaning at one end and dissemination of information at the other. After the prosperous development and reproduction of systems and software that are able to efficiently and effectively store, retrieve, and process data, attentiveness has now shifted to predictive and correlative analytics [5]. Machine learning and data science fields achieved strong demonstration in perdition, this demand on a unique mindset, one that has heretofore seen some representation in academic curriculum and in the literature of social science [6].

To employ data science in education, students can receive information about their performance in relation to their colleagues or about their improvement in relation to their goals can be encouraging and motivating. On the other hand, administrators and decision-makers can take the benefits of data science to strive to improve the educational environment.

The main contribution of this paper can be summarized as follows: Machine Learning is widely used to predict the performance of students in an academic institution. Several ML algorithms are employed where several different attributes are addressed and considered for students' performance prediction. In this article, the capability of employing the CNN deep learning algorithm is addressed and exanimated in the area of predicting students' achievements, where several parameters are manipulated to produce the best performance result in terms of Recall, Precision, F-measures. The work presented in this paper produce promising results in comparison with few related works results.

The possibility of using huge amounts of data for decision-making became feasible in the 80s. The field of Data Mining flourished in the early 90s as database technologies and business processes became automated. Many books on Data Mining from the 90s described how Machine Learning methods could be applied to a solve many of business problems. There was an identical explosion in the obtainable software tools prepared towards leveraging behavioral and transactional data for explanation and prediction purposes [6].

Authors [7] highlighted the data science as the copulative tissue between data-processing technologies and data-driven decision-making. In addition, they discussed the complex issue of data science as a profession versus data science as a field. Many Previous researches presented various types of analytics and how it can be used in the academic fields, [8] proposed a conceptual framework that characterize the types of analytics and their relationship to each other. In addition, they proposed a synthesized set of analytics-related terms generally found in academic field.

Data Mining is a concept depends on extracting hidden pattern and discovering the relationships between parameters in amount of data. Authors in [9] proposed a framework for predicting students' performance by using Naïve Bayes, Decision Tree, and Rule Based classification techniques in order to produce the best students' performance prediction model, the result proof that the Rule Based is the best model among the other classification techniques by achieving the highest accuracy values.

Two experiments are conducted using data from high school Mexican students. Authors noticed that most of the current research on EDM Applications to solve the problems of courses dropping out by student is addressed for determined cases of higher education. The research into mandatory education dropout rates has been conducted and found that the proposed work discovers trustworthy classification models to help students make an early decision of dropout, before the middle of the course [10].

Several researchers concentrate on the student performance through their educational journey. Chanintorn [11] tries to develop a system that employs data from the LMS to help in classifying student preferences and personalities. This model aims to build new curricula that might suit student personalities, in order to support them to be more effective and efficient in their study.

Abdul Munem [12] explores the effectiveness of employing educational blogs in teaching computers materials on students' college performance and their retention capability. Several grouped are addressed and different factors are investigated. SPSS is used to validate the hypotheses in his study.

Recently, the big companies' success is constructed with the rise of the Big data science and the success of deep learning (DL). Using DL is no limited to be used in Cyber-security, health industry, retail, Banking and robotics etc. Priyanka Patel and Amit Thakkar [13] conclude that DL systems start smash most classical ways, but also, human benchmarks in numerous tasks like image classification, action detection, natural language processing, signal process, and linguistic communication process. Deep learning algorithms are widely used in several domains for classification and prediction purposes. 
Students' performance prediction is one of the hot fields where DL is used to predict and enhance the student performance in the academic field.

An effort is made to predict students' success by collecting students' data from several Pakistani universities. Set of classification models and Learning analytics are utilized to expect if the studied student will pursue his bachelor degree or not. Research results show that the developed method outperforms previous methods due to students' personal information feature sets [2].

Authors in [3] conducted a systematical literature review to predict student achievement by utilizing data mining techniques in order to improve student's performance. The proposed research provided an overview on the data mining techniques that have been used in students' performance prediction. This research also focused on how the prediction algorithms can be utilized to identify the main attributes in a student's data.

A Data Science Machine is developed by [5], which automatically extract predictive models from raw data. Authors entered the Data Science Machine in three data science competitions that featured other data science teams. The proposed approach compete previous teams in these data science competitions. In tow of the three competitions they compete a many of competitors, and in the third, they achieved high percent score.

Deep learning suppose that complex functions may be built by restructuring simple functions like Gaussian kernels, Deep learning is composed of multiple 2-layer sequences, feature pooling layer and feature detection layer, where in each layer a supervision step is executed [14]. Authors in [15] used Deep learning in a supervised manner and unsupervised manner to develop prediction models with given inputs and output, and to extract useful features from raw data, such as online services they rely on machine learning to extract valuable information from data collected in the web[16]. Authors in [17] reviewed some issues that face deep learning such as black-box models, unsupervised learning, and online learning to clarify how these challenges can be used into productive investment in future research approaches.

In recent machine learning developed researches, there are more developed techniques to represent the complex entities. In [18] they trained an embedded program submission sequence on two tasks of predicting the student's future performance, while authors in [19] proposed a new deep learning algorithm based on examining the student performance prediction issue as a sequential event prediction issue. A study developed by [20] focused on how student will perform at the end of a course, indicated that the integration of behavioral features and textual features are more predictive than only behavioral features, in [21] Students from three colleges were considered in the research, which students records were applied on deep learning. The results were compared with other classification methods, and the authors have found that the highest classification rate produced by the deep learning techniques.

However, deep learing techneques have aslo been used in predicting individual achievements. Proposed a personalized model to detect a suitable learning techneque based on a deep learningalgorithm [22]. In [23] provided a general review of deep learning techniques that are developed to predict the student's performance, and they developed a model that effectively predict the student GPA by using machine learning techniques. While the study in [24] intended to support institutions in formulating an essential framework for pedagogical support, and facilitating educational decision-making processes towards sustainable educational development goals.

\section{RESEARCH METHOD}

\subsection{Dataset}

The data set used in this paper is taken from a learner activity tracker tool [25] experience API (xAPI). The xAPI provides learning and training architecture (TLA) that facilitate tracking the learning improvement and monitoring the learner's activities such as writing an article, watching a video or reading article etc. The xAPI provides the learning platform facilitator to identify the learner, actions and all related elements that might help in addressing the learning practice.

The dataset consists of 480 student records and 16 attributes. These attributes are categorized into three main classifications: (1) Demographic related attributes like gender and nationality. (2) Academic related attributes like educational stage, grade Level and section. (3) Behavioral related attributes like hand rising in classes, opening resources, doing surveys with classmates, and school satisfaction.

The dataset consists of the two genders 305 and 175 male and females respectively. This data set collects all information about the participated students, those students belong to different country, for example 179 participated students from Kuwait, 172 participated students from Jordan, 28 participated students from Palestine, 22 students from Iraq, 17 students from Lebanon, 12 students from Tunis, 11 students from Saudi Arabia, 9 students from Egypt, 7 students from Syria, 6 students from USA, Iran and Libya, 4 students from Morocco and 1 student from Venezuela. The dataset is collected through 2 academic 
terms: in the first term, the total numbers of collected data are recorded for 245 students while in the second semester; the total numbers of collected data are recorded for 235.

The data set also collect student's attendance attribute, as they found in total 191 of the participated students get absent for more than 7 days and 289 of the participated students have less than 7 days of absences. This dataset does not ignore the crucial role of the students' parents in the learning and achievements progress. The students' parents participate as well in this data set by surveying them in regards with their satisfaction and all related factors to the educational process. Data set attributes as shown in Table 1.

Table 1. Data set attributes

\begin{tabular}{cc}
\hline Attribute name & Description \\
\hline Gender & student's gender \\
Nationality & All participated student's country \\
POB & All participated student's place of birth country \\
Educational Stages & The Educational levels of the participated students \\
Grade Levels & The Grade levels of the participated students \\
Section ID & participated student's Section number \\
Topic & The course area \\
Semester & The academic term \\
Parent responsible for student & The participated student's associated parent gender \\
Raised hand & Total number of raising hand per semester (numeric:0-100) \\
Visited resources & Total number of visiting course material \\
Viewing announcements & Total number of reading announcements \\
Discussion groups & Total number of discussion participation \\
Parent Answering Survey & Total number of surveys that have been answered by parent \\
Parent School Satisfaction & School satisfaction from parent perspectives \\
Student Absence Days & Total number of participated students absences \\
\hline
\end{tabular}

\subsection{Research Steps}

CNN is widely used for prediction purposes, as CNN provide several settings which have to be defined and manipulated to reach the optimal performance accuracy. These factors have high impact on the performance of the CNN model (i.e. activation function, number of layers, number of neuron in each layer and hyper-parameter).

Figure 1 reveals the main steps in the research methodology. In the proposed research methodology, after the data set is collected, set of experiments will be conducted to train the model and other will be used to test the proposed model. Authors adopt the number of epoch and number of layers as main factors to manipulate until the optimal accuracy will be achieved.

As a result of the experiments the reached accuracy measures will be compared with few works in the literature who's addressed the performance prediction of students' achievement. Figure summarizes the main step of the proposed research.

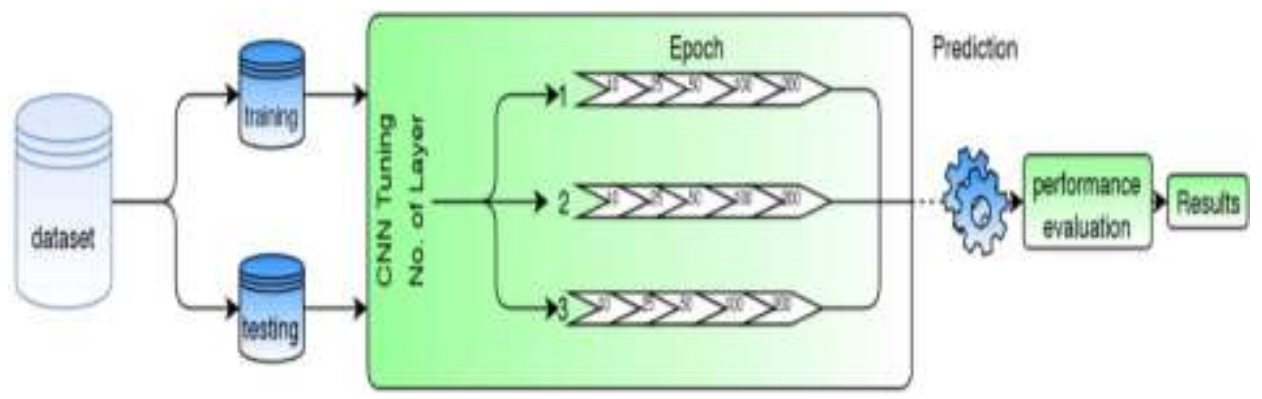

Figure1. Methodology steps

\section{RESULTS AND ANALYSIS}

As a baseline structure, The CNN (Convolutional neural network) is composed of Convolution 1D layer, pooling 1D layer, fully connected layer, and ReLU non-linear activation function. Different parameters for CNN structure have been changed to see their impact on the prediction. To identify the CNN network structure for distinguishing results, the following experiments are performed for the aforementioned network 
topologies. Mainly, the experiments considered different number of epoch with different number of layer to check the best result in term of accuracy and F-measures. Moreover, the precision and the recall measures are calculated.

Table 2-6 show how the experiments are producing better result in term of accuracy by increasing the number of epoch. Author adopt three level of layer for comparison and for each experiment the number of epoch is increased gradually from 10-200 which produced the best accuracy so only five main experiments are performed while in real 15 experiments are conducted as the number of layers is manipulated as well from 1-3 for each experiments. Obviously, experiment number five achieves the highest prediction accuracy when the number of epoch is 200 with number of layers 2 and three.

Table 2. Number of epoch (10)

\begin{tabular}{ccccc}
\hline \multicolumn{5}{c}{ CNN's Results } \\
No of layer & Accuracy & Precision & Recall & F-measure \\
\hline ONE & .594 & .468 & .594 & .509 \\
TWO & .620 & .620 & .620 & .613 \\
THREE & .575 & .687 & .575 & .499 \\
\hline
\end{tabular}

Table 4. Number of epoch (50)

\begin{tabular}{ccccc}
\hline \multicolumn{5}{c}{ CNN's Results } \\
No of layer & Accuracy & Precision & Recall & F-measure \\
\hline ONE & .639 & .675 & .639 & .641 \\
TWO & .981 & .981 & .981 & .980 \\
THREE & 1.00 & 1.00 & 1.00 & 1.00 \\
\hline
\end{tabular}

Table 3. Number of epoch (25)

\begin{tabular}{ccccc}
\hline \multicolumn{5}{c}{ CNN's Results } \\
No of layer & Accuracy & Precision & Recall & F-measure \\
\hline ONE & .626 & .629 & .690 & .579 \\
TWO & .829 & .832 & .829 & .827 \\
THREE & .892 & .909 & .892 & .885 \\
\hline
\end{tabular}

Table 5. Number of epoch (100)

\begin{tabular}{ccccc}
\hline \multicolumn{5}{c}{ CNN's Results } \\
No of layer & Accuracy & Precision & Recall & F-measure \\
\hline ONE & .816 & .819 & .816 & .817 \\
TWO & .993 & .993 & .993 & .993 \\
THREE & 1.00 & 1.00 & 1.00 & 1.00
\end{tabular}

Table 6. Number of epoch (200)

\begin{tabular}{ccccc}
\hline \multicolumn{5}{c}{ CNN's Results } \\
No of layer & Accuracy & Precision & Recall & F-measure \\
\hline ONE & .955 & .956 & .955 & .955 \\
TWO & 1.00 & 1.00 & 1.00 & 1.00 \\
THREE & 1.00 & 1.00 & 1.00 & 1.00 \\
\hline
\end{tabular}

Table 7 summarizes a short comparison with few researches that have been done recently in the studied area. As shown in Table 7, the best result was achieved by [10] as they employed NaiveBayes algorithm and their result accomplished $89 \%$ in terms of accuracy. The proposed approach in our paper outperforms the prediction performance of the studied related works. Moreover, several measurements, not just recall, were calculated to provide comprehensive prediction performance perspectives to the readers.

Table 7. Related work Results

\begin{tabular}{ccc}
\hline Reference & Method used & Accuracy \\
\hline$[9]$ & Rule Based (RB) & 0.713 \\
{$[9]$} & Naïve Bayes (NB) & 0.67 \\
{$[9]$} & DT decision tree & 0.688 \\
{$[2]$} & SVM & 0.867 \\
{$[10]$} & Support Vector & 0.8723 \\
& Machines & \\
{$[10]$} & NaiveBayes & 0.89 \\
{$[10]$} & Class Association Rules CAR & 0.80 \\
\hline
\end{tabular}

\section{CONCLUSION}

This paper addressed the capability of employing the CNN deep learning algorithm in the area of predicting students' achievements. Several parameters were manipulated to produce the result performance in terms of Recall, Precision, F-measures. The work presented in this paper produce promising results in comparison with few related works results. As future works, authors will seek to find bigger data set then implement and employ more deep learning algorithms to address their effectiveness in the studied area. 


\section{REFERENCES}

[1] Daniel, Ben, "Big Data and analytics in higher education: Opportunities and challenges," British journal of educational technology, vol. 46, no. 5, pp. 904-920, 2015.

[2] Daud, Ali, et al., "Predicting student performance using advanced learning analytics," Proceedings of the 26th international conference on world wide web companion. International World Wide Web Conferences Steering Committee, 2017.

[3] Shahiri, Amirah Mohamed, and Wahidah Husain, "A review on predicting student's performance using data mining techniques," Procedia Computer Science vol. 72, pp.: 414-422, 2015.

[4] Van Der Aalst, Wil, "Data science in action," Process Mining. Springer, Berlin, Heidelberg, 2016. 3-23.

[5] Kanter, James Max, and Kalyan Veeramachaneni, "Deep feature synthesis: Towards automating data science endeavors," IEEE International Conference on Data Science and Advanced Analytics (DSAA), pp. 1-10, 2015.

[6] Dhar, Vasant, "Data Science and Prediction (October 2012)," NYU Working Paper No. 2451/31635, [Online] Available : at SSRN https://ssrn.com/abstract=2071041

[7] Provost, Foster, and Tom Fawcett, "Data science and its relationship to big data and data-driven decision making," Big data vol. 1, no. 1, pp. 51-59, 2013.

[8] Van Barneveld, Angela, Kimberly E. Arnold, and John P. Campbell, "Analytics in higher education: Establishing a common language," EDUCAUSE learning initiative vol. 1, no. 1, pp. 1-11., 2012.

[9] Ahmad, Fadhilah, Nur Hafieza Ismail, and Azwa Abdul Aziz, "The prediction of students' academic performance using classification data mining techniques," Applied Mathematical Sciences, vol. 9, no. 129, pp. 6415-6426, 2015.

[10] Márquez-Vera, Carlos, et al., "Early dropout prediction using data mining: a case study with high school students," Expert Systems, vol. 33, no. 1, pp. 107-124, 2016.

[11] Jittawiriyanukoon, Chanintorn, "Proposed classification for elearning data analytics with MOA," International Journal of Electrical \& Computer Engineering, vol. 9, pp. 2088-8708, 2019.

[12] Abdul Munem, "Effectiveness of the use of electronic educational blogs in teaching computers on the achievement of students," Indonesian Journal of Electrical Engineering and Computer Science, vol. 17, no. 1, pp. 489 499, January 2020.

[13] Priyanka Patel, Amit Thakkar, "The upsurge of deep learning for computer vision applications," Indonesian Journal of Electrical Engineering and Computer Science, vol. 10, no.1, pp. 538-548, February 2020.

[14] Bengio, Yoshua, and Yann LeCun, "Scaling learning algorithms towards AI," Large-scale kernel machines, vol. 34, no. 5, pp. 1-41, 2007.

[15] Fan, Cheng, Fu Xiao, and Yang Zhao, "A short-term building cooling load prediction method using deep learning algorithms," Applied energy, vol. 195, pp. 222-233, 2017.

[16] Muñoz-González, Luis, et al., "Towards poisoning of deep learning algorithms with back-gradient optimization," Proceedings of the 10th ACM Workshop on Artificial Intelligence and Security, 2017.

[17] Pouyanfar, Samira, et al., "A survey on deep learning: Algorithms, techniques, and applications," ACM Computing Surveys (CSUR) vol. 51, no. 5, pp. 1-36, 2018.

[18] Wang, Lisa, et al., "Learning to Represent Student Knowledge on Programming Exercises Using Deep Learning," International Educational Data Mining Society, 2017.

[19] Kim, Byung-Hak, Ethan Vizitei, and Varun Ganapathi, "GritNet: Student performance prediction with deep learning," arXiv preprint arXiv: 1804.07405, 2018.

[20] Tu, Yuwei, Weiyu Chen, and Christopher G. Brinton, "A Deep Learning Approach to Behavior-Based Learner Modeling," arXiv preprint arXiv:2001.08328, 2020.

[21] Hussain, Sadiq, et al., "Prediction model on student performance based on internal assessment using deep learning," International Journal of Emerging Technologies in Learning (iJET), vol. 14, no. 08 pp. 4-22, 2019.

[22] Mansur, Andi Besse Firdausiah, Norazah Yusof, and Ahmad Hoirul Basori, "Personalized Learning Model based on Deep Learning Algorithm for Student Behaviour Analytic," Procedia Computer Science vol. 163, pp. 125-133, 2019.

[23] Patil, Akhilesh P., Karthik Ganesan, and Anita Kanavalli, "Effective deep learning model to predict student grade point averages," IEEE International Conference on Computational Intelligence and Computing Research (ICCIC), pp. 1-6, 2017.

[24] Waheed, Hajra, et al., "Predicting academic performance of students from VLE big data using deep learning models," Computers in Human Behavior, vol. 104, pp. 106189, 2020.

[25] Students' Academic Performance Dataset, [Online], available https://www.kaggle.com/aljarah/xAPI-Edu-Data, Access on August 2019. 


\section{BIOGRAPHIES OF AUTHORS}

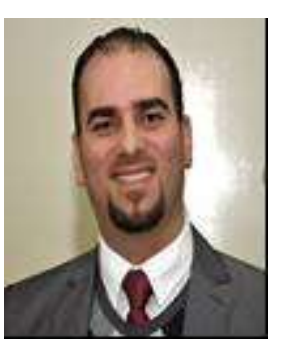

Dr. Mohammed Akour is an associate Professor of Software Engineering at Al Yamamah University (YU). He got his Bachelor's (2006) and Master's (2008) degree from Yarmouk University in Computer Information Systems with Honor. He joined Yarmouk University as a Lecturer in August 2008 after graduating with his master's in Computer Information Systems. In August 2009, He left Yarmouk University to pursue his Ph.D. in Software Engineering at North Dakota State University (NDSU). He joined Yarmouk University again in April 2013 after graduating with his Ph.D. in Software Engineering from NDSU with Honor. He serves as Keynote Speaker, Organizer, a Co-chair and publicity Chair for several IEEE conferences, and as ERB for more than 10 ISI indexed prestigious journals. $\mathrm{He}$ is a member of the International Association of Engineers (IAENG). Dr. Akour at Yarmouk University served as Head of accreditation and Quality assurance and then was hired as director of computer and Information Center. In 2018, Dr. Akour has been hired as Vice Dean of Student Affairs at Yarmouk University. In 2019, Dr. Akour joins Al Yamamah University -Riyadh Saudi Arabia- as an associate professor in Software Engineering.

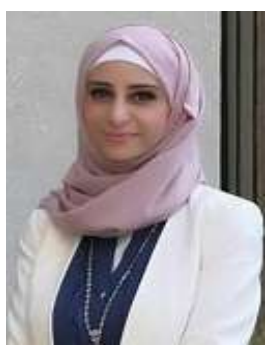

Hiba Alsghaier got her Master's degree in Computer Information Systems from the Faculty of Information Technology and Computer Sciences, Yarmouk University. She worked at Jordan University of Science Technology as a teaching assistant for a few years, and now she is a web designer and a member in statistical consulting center advisory board at JUST, Alsghaier has many publications in the fields of software engineering, big data analytics, and software fault prediction and currently she is working on other field

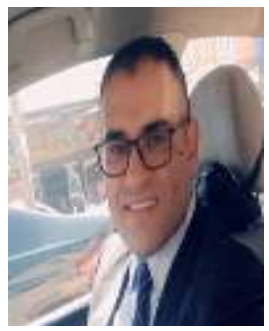

Osama Al Qasem got his Master's degree in Computer Information Systems from the Faculty of Information Technology and Computer Sciences, Yarmouk University. Al Qasem Published two articles in the fields of big data analytics, and software fault prediction. 